\title{
Same-sign top quarks as signature of light stops ${ }^{1}$
}

\author{
Sabine Kraml* and Are R. Raklev ${ }^{*, \dagger}$ \\ ${ }^{*}$ Theory Division, Dept. of Physics, CERN, CH-1211 Geneva 23, Switzerland \\ ${ }^{\dagger}$ Dept. of Physics and Technology, University of Bergen, N-5007 Bergen, Norway
}

\begin{abstract}
We present a new method to search for a light scalar top (stop), decaying dominantly into $c \tilde{\chi}_{1}^{0}$, at the LHC. The principal idea is to exploit the Majorana nature of the gluino, leading to same-sign top quarks in events of gluino pair production followed by gluino decays into top and stop. We demonstrate the reach of our method in terms of the gluino mass and the stop-neutralino mass difference.
\end{abstract}

Keywords: Supersymmetry; Hadron colliders

PACS: $12.60 . J v, 14.65 . \mathrm{Ha}, 14.80 . \mathrm{Ly}$

\section{INTRODUCTION}

If the lighter of the two stops, $\tilde{t}_{1}$, has mass $m_{\tilde{t}_{1}} \lesssim m_{t}$, as motivated by electroweak baryogenesis (EWBG) in the MSSM [1, 2, 3, 4, 5], gluino decays into stops and tops will have a large branching ratio. Since gluinos are Majorana particles, their decays do not distinguish between $t \tilde{t}_{1}^{*}$ and $\tilde{t}_{1}$ combinations. Pair-produced gluinos therefore give

$$
\tilde{g} \tilde{g} \rightarrow t \bar{t} \tilde{t}_{1} \tilde{t}_{1}^{*}, t t \tilde{t}_{1}^{*} \tilde{t}_{1}^{*}, \bar{t} \tilde{t} \tilde{t}_{1} \tilde{t}_{1}
$$

and hence same-sign top quarks in half of the gluino-to-stop decays. If the stopneutralino mass difference is small, $m_{\tilde{t}_{1}}-m_{\tilde{\chi}_{1}^{0}}<m_{W}$, the stop may further decay into $c \tilde{\chi}_{1}^{0}$. A mass difference in the range $10-30 \mathrm{GeV}$ can, in fact, provide the correct dark matter abundance in stop-coannihilation scenarios [6, 7]. If, in addition, the $W$ stemming from $t \rightarrow b W$ decays leptonically, this gives a signature of two $b$-jets plus two same-sign leptons plus jets plus missing transverse energy:

$$
p p \rightarrow \tilde{g} \tilde{g} \rightarrow b b l^{+} l^{+}\left(\text {or } \bar{b} \bar{b} l^{-} l^{-}\right)+\text {jets }+\not L_{T} .
$$

This is a quite peculiar signature, which will serve to remove most backgrounds, both SM and SUSY. Even though stop pair production has the dominant cross section, it leads to a signature of two $c$-jets and missing transverse energy, which is of very limited use at the LHC. Thus the same-sign top signature is of particular interest in our scenario.

To investigate the use of Eq. (2) for discovering a light stop at the LHC, we defined in [8] an MSSM benchmark point 'LST1' with $m_{\tilde{t}_{1}}=150 \mathrm{GeV}$. For simplicity the other squarks were taken to be heavier than the gluinos, considerably suppressing the SUSY background, with gluinos decaying to $100 \%$ into $t \tilde{t}_{1}$. The MSSM parameters of LST1 can be found in [8]. We list the corresponding masses in Table 1

\footnotetext{
${ }^{1}$ Talk given by ARR at SUSY06, Irvine, California, USA, 12-17 June 2006.
} 
TABLE 1. SUSY mass spectrum [in GeV] for the LST1 scenario. For the squarks and sleptons, the first two generations have identical masses.

\begin{tabular}{cccccccc}
\hline Particle & Mass & Particle & Mass & Particle & Mass & Particle & Mass \\
\hline$\tilde{d}_{L}$ & 1001.69 & $\tilde{u}_{L}$ & 998.60 & $\tilde{b}_{1}$ & 997.43 & $\tilde{t}_{1}$ & 149.63 \\
$\tilde{d}_{R}$ & 1000.30 & $\tilde{u}_{R}$ & 999.40 & $\tilde{b}_{2}$ & 1004.56 & $\tilde{t}_{2}$ & 1019.26 \\
$\tilde{e}_{L}$ & 254.35 & $\tilde{\tau}_{1}$ & 247.00 & $\tilde{v}_{e}$ & 241.90 & $\tilde{v}_{\tau}$ & 241.90 \\
$\tilde{e}_{R}$ & 253.55 & $\tilde{\tau}_{2}$ & 260.73 & & & & \\
$\tilde{g}$ & 660.00 & $\tilde{\chi}_{1}^{ \pm}$ & 188.64 & $\tilde{\chi}_{2}^{ \pm}$ & 340.09 & & \\
$\tilde{\chi}_{1}^{0}$ & 104.81 & $\tilde{\chi}_{2}^{0}$ & 190.45 & $\tilde{\chi}_{3}^{0}$ & 306.06 & $\tilde{\chi}_{4}^{0}$ & 340.80 \\
$h$ & 118.05 & $H$ & 251.52 & $A$ & 250.00 & $H^{ \pm}$ & 262.45 \\
\hline
\end{tabular}

Using a fast simulation of a generic LHC detector we found in [8] that our signal could easily be separated from the background at the LST1 benchmark point with $30 \mathrm{fb}^{-1}$ of integrated luminosity. We further showed that a relationship between the neutralino, stop and gluino masses could be found. Here we demonstrate the reach of our method in terms of the gluino mass and stop-neutralino mass difference by performing a scan for the signal significance of Eq. (2) over a large set of parameter choices.

\section{SIMULATION AND RESULTS}

\section{Event generation and signal isolation}

For the LST1 benchmark point we found a large signal significance ${ }^{2}$ of $80 \sigma$ with the gluino-pair cross section fairly evenly divided between direct pair production and gluino production from the decay of squarks. In the presence of CP violation in the chargino and neutralino sectors, as required for successful EWBG in the MSSM, the constraints from electric dipole moments are often evaded by making the first and second generations of squarks and sleptons very heavy. In this contribution, we hence set all sfermion masses, except the stop, to $10 \mathrm{TeV}$ while the chargino and neutralino parameters are kept as in LST1. This removes the squark contribution to the inclusive gluino-pair cross section and leaves us with the gluino and light-stop masses as free parameters.

The signal cross section is given in Table 2 as a function of the gluino mass. We showed in [8] that after reasonable cuts only a handful of SUSY background events remained, all resulting from squark decays. Here we can ignore this background source, because of our choice of very heavy squarks, and only consider the SM background, where in particular the top pair production is important. It is thus sufficient to generate $30 \mathrm{fb}^{-1}$ of gluino pair production in a scan over 60 points, using PYTHIA 6.321 [10] and CTEQ 5L [11] parton distribution functions. Details of the SM background sample and the detector simulation carried out with AcerDET 1.0 [12] can also be found in [8].

\footnotetext{
${ }^{2}$ We define significance as $S / \sqrt{B}$, where $S$ and $B$ are the numbers of signal and background events.
} 
TABLE 2. The NLO gluino pair production cross sections computed with PROSPINO2 [9]. This is insensitive to the exact squark masses down to the $1 \%$ level.

\begin{tabular}{lcccccccccc}
\hline$m_{\tilde{g}}[\mathrm{GeV}]$ & 300 & 400 & 500 & 600 & 700 & 800 & 900 & 1000 & 1100 & 1200 \\
$\sigma(\tilde{g} \tilde{g})[\mathrm{pb}]$ & 535 & 113 & 31.6 & 10.4 & 3.84 & 1.56 & 0.68 & 0.31 & 0.20 & 0.10 \\
\hline
\end{tabular}
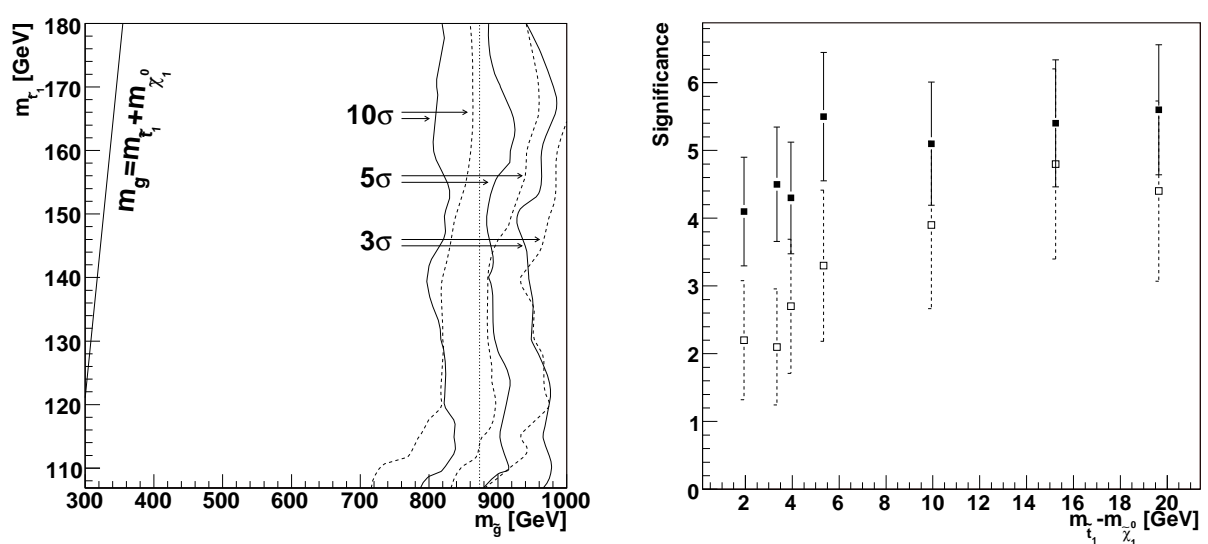

FIGURE 1. Reach for the signature of Eq. (2) in the gluino-stop mass plane (left) and significance as a function of stop-neutralino mass difference with $m_{\tilde{g}}=900 \mathrm{GeV}$ (right), for the two sets of cuts considered in the text: Cuts I (dashed lines) and Cuts II (solid lines).

In the following we compare two sets of cuts. Cuts I are the cuts used in [8], to identify events with the signature of Eq. (2), in addition to standard cuts in the search for SUSY:

- Require two same-sign leptons $\left(e\right.$ or $\mu$ ) with $p_{T}^{\text {lep }}>20 \mathrm{GeV}$.

- Require at least four jets with $p_{T}^{\text {jet }}>50 \mathrm{GeV}$, at least two of which are $b$-tagged.

- $E_{T}>100 \mathrm{GeV}$.

- Demand two combinations of the two hardest leptons and $b$-jets that give invariant masses $m_{b l}<160 \mathrm{GeV}$, consistent with a top quark.

The other set of cuts, Cuts II, are identical, except that we relax the requirement of four jets to only two $b$-tagged jets. This set of cuts emphasizes the role of the same-sign top quarks in our method, and ignores the detectability of the jets initiated by the $c$-quarks.

\section{Results}

In Fig. 1 (left) we show the reach of the signature in Eq. (2) for the two sets of cuts by plotting contours of $10 \sigma, 5 \sigma$ and $3 \sigma$ significance (from left to right). Despite the fluctuations due to large statistical errors on the calculated significance, we see clear trends. Cuts I give larger significance from better rejection on background at higher stop masses, i.e. larger stop-neutralino mass differences, because of the added requirement of seeing the jets from the $c$-quarks. At low mass difference, Cuts II perform better just 
because they ignore these soft jets. For comparison we also show (dotted line) the results of a recent full simulation study for the CMS detector [13], which found a reach down to $1 \mathrm{pb}$ in terms of the total cross section for same-sign top production.

Figure 1(right) shows the decreasing significance for $m_{\tilde{g}}=900 \mathrm{GeV}$ and for both sets of cuts, as the stop-neutralino mass difference goes to zero. Note that Cuts I do not have a total loss of signal as might naively be expected. This is due to erroneously picking ISR, FSR or jets from the underlying event, as the $c$-quark jets. For these small mass differences, neutralino-stop coannihilation is very efficient. In the scenario studied here, a neutralino relic density of $\Omega_{\chi} h^{2} \simeq 0.1$ is obtained for $m_{\tilde{t}_{1}}-m_{\tilde{\chi}_{1}^{0}} \sim 20 \mathrm{GeV}$ [14, 15].

\section{CONCLUSIONS}

We have investigated baryogenesis and dark-matter motivated scenarios with a light stop, $m_{\tilde{t}_{1}} \lesssim m_{t}$, with $\tilde{t}_{1} \rightarrow c \tilde{\chi}_{1}^{0}$ as the dominant decay mode. In gluino pair production, the Majorana nature of the gluino leads to a peculiar signature of same-sign top quarks in half of the gluino-to-stop decays. We have shown that with $30 \mathrm{fb}^{-1}$ of data the search for such a signature at the LHC has a reach in terms of gluino mass of up to $m_{\tilde{g}} \sim 900 \mathrm{GeV}$, even for stop-neutralino mass differences in the stop-coannihilation region.

\section{ACKNOWLEDGMENTS}

We thank Marcela Carena and Stefano Profumo for helpful discussions. SK is supported by an APART (Austrian Programme of Advanced Research and Technology) grant of the Austrian Academy of Sciences. ARR acknowledges support from the European Community through a Marie Curie Fellowship for Early Stage Researchers Training.

\section{REFERENCES}

1. D. Delepine, J. M. Gerard, R. Gonzalez Felipe, and J. Weyers, Phys. Lett. B386, 183-188 (1996), hep-ph/9604440.

2. M. Carena, M. Quiros, and C. E. M. Wagner, Nucl. Phys. B524, 3-22 (1998), hep-ph/9710401

3. J. M. Cline, and G. D. Moore, Phys. Rev. Lett. 81, 3315-3318 (1998), hep-ph/980 6354

4. C. Balazs, M. Carena, and C. E. M. Wagner, Phys. Rev. D70, 015007 (2004), hep-ph/ 0403224

5. V. Cirigliano, S. Profumo, and M. J. Ramsey-Musolf, JHEP 07, 002 (2006), hep-ph/ 0603246

6. C. Boehm, A. Djouadi, and M. Drees, Phys. Rev. D62, 035012 (2000), hep-ph/9911496.

7. J. R. Ellis, K. A. Olive, and Y. Santoso, Astropart. Phys. 18, 395-432 (2003), hep-ph/ 0112113

8. S. Kraml, and A. R. Raklev, Phys. Rev. D73, 075002 (2006), hep-ph/0512284

9. W. Beenakker, R. Hopker, and M. Spira (1996), hep-ph/9611232

10. T. Sjostrand, et al., Comput. Phys. Commun. 135, 238-259 (2001), hep-ph/0010017

11. H. L. Lai, et al., Eur. Phys. J. C12, 375-392 (2000), hep-ph/9903282

12. E. Richter-Was (2002), hep-ph/0207355.

13. CMS Collaboration, CMS Physics: Technical Design Report, Volume 2: Physics Performance (2006), CMS-TDR-8.2, CERN-LHCC-2006-021.

14. C. Balazs, M. Carena, A. Menon, D. E. Morrissey, and C. E. M. Wagner, Phys. Rev. D71, 075002 (2005), hep-ph/0412264

15. G. Belanger, F. Boudjema, S. Kraml, A. Pukhov, and A. Semenov, Phys. Rev. D73, 115007 (2006), hep-ph/0604150 